\title{
新工科视角下分析化学类课程科研型教学模式的构建与实践
}

卢松涛, 王群, 李杨, 姚远, 康红军, 吴晓宏 ${ }^{*}$

哈尔滨工业大学化工与化学学院, 哈尔滨 150001

\begin{abstract}
摘要: 基于 “新工科” 建设背景, 哈尔滨工业大学分析化学类课程积极构建科研型教学模式。根据授课各专业的特点结 合最新科研进展更新教学内容, 打通课堂教学与科研成果的通道, 改进教学方法, 更新实践教学资源, 开放表界面材料 分析化学实验室, 以学科竞赛及创新创业项目为载体, 激发学生创新思维, 开阔学生国际视野。
\end{abstract}

关键词: 新工科; 分析化学; 科学研究; 教学改革

中图分类号: O6; G64

\section{Construction and Practice of Scientific Research Teaching Mode of Analytical Chemistry Course in the View of Emerging Engineering Education}

\author{
Songtao Lu, Qun Wang, Yang Li, Yuan Yao, Hongjun Kang, Xiaohong Wu * \\ School of Chemistry and Chemical Engineering, Harbin Institute of Technology, Harbin 150001, China.
}

\begin{abstract}
Based on the background of "emerging engineering education" construction, some methods were employed for the analytical chemistry course in Harbin Institute of technology, including updating the teaching content according to the characteristics of each major, combining with the latest scientific research progress in order to open the channel between classroom teaching and scientific research results, improving teaching skills, enriching practical teaching resources, opening the surface and interface material analytical chemistry laboratory, and stimulating learning interests by discipline competition and innovation and entrepreneurship projects as carriers. These methods can further improve teaching quality and broaden students' international vision, and comprehensively improve students' innovative spirit and ability.
\end{abstract}

Key Words: Emerging engineering education; Analytical chemistry; Scientific research; Teaching reform

作为人类社会发展的重要引擎, 新一轮的世界科技革命和产业变革亟需新型工程技术人才。近 年来, 我国积极推动 “新工科” 建设, 创新多元化、实践性与国际化是新工科的教育方式特色。“新 工科” 人才具有工程实践能力强、创新能力强和高国际竞争力, 以及快速学习新事物的能力, 具备 全球视野、领导能力和实践能力 ${ }^{[1]}$ 。2019年10月, 教育部印发《关于深化本科教育教学改革全面提高 人才培养质量的意见》, 提出要推动科研反哺教学, 强化科研育人功能, 激发学生专业学习兴趣。

哈尔滨工业大学分析化学类课程包括分析化学、仪器分析、薄膜材料制备与技术, 它们作为材 料、环境、食品、生命科学等相关专业的平台课程, 是各专业必修的一门基础课。它主要研究元素

收稿: 2020-11-24; 录用: 2020-12-04; 网络发表: 2020-12-28

“通讯作者, Email: wuxiaohong@hit.edu.cn

基金资助: 中国建设教育协会课题(2019083) 
及其化合物的性质、常用的分析仪器基本原理和定量分析方法的基本操作。现代科学的最新研究进 展极大地丰富了分析化学内容, 传统的分析化学教学只关注学生对于基础知识的掌握和对基本操作 技能的培训, 而无法满足现代社会对 “新工科” 人才创新思维模式的需求 ${ }^{[2,3]}$ 。本课程团队教师均有 一年以上的国外教学和科研访学经历, 包括在美国大学分析化学排名第五的德克萨斯大学奥斯汀分 校, 排名第六的威斯康星大学麦迪逊分校, 杜克大学和新加坡国立大学, 了解国际分析化学领域研 究现状, 课题紧密围绕表界面功能薄膜材料的分析化学领域发展趋势, 在 “空间用材料分析化学” 领域的研究方面形成优势与特色。如何根据各授课专业的特点结合最新科研进展更新教学内容, 教 学与科研成果融合, 改进教学方法, 更新实践教学资源, 进一步提高教学质量, 是任课教师十分关 心和亟待解决的重要课题。

\section{1 国内外研究现状分析}

\section{1 国内研究现状分析}

目前中国高校的分析化学课程大多是以教师为主的满堂灌授课方式。教学模式陈旧, 过于单一、 缺乏创新思维。教师需要将学生集中起来, 统一讲授实验测试技术原理、目的、仪器及试剂的使用, 以及实验过程中的具体注意事项, 由于大学生在整个课程学习过程中基本上处于盲目接收知识的被 动状态, 没有时间和机会积极创造性思维, 限制了学生扩散性思维及批判性思维的培养, 容易减弱 学生的好奇心, 整个过程中几乎没有创新思维的植入, 对于人才培养是及其不利的。

实验内容缺乏新意, 脱离实际应用。学生被动地接受各种各样的知识和信息, 没有时刻更新内 容和新进展。部分高校对大学生培养过程中的分析化学实践环节没有引起足够重视, 创新能力明显 不足, 缺乏原创性、实用性的教学实验资源, 使得学生的实验能力跟不上企业或是最新的科学研究 工作。学生不会主动进行文献调研, 没有自己的想法, 导师让做什么实验, 学生就做什么, 人云亦 云, 在实验方法上简单移植, 在实验过程中仅简单揭示表面现象, 无法独立深入挖掘科学问题等。

\section{2 国外分析化学课程现状}

国外著名大学如麻省理工学院、加州大学、剑桥大学等欧美大学分析化学课教学模式多种多样, 上课主要讲授重点、难点、习题等, 突出课程内容的国际性、前沿性、研究性和创新性。将前沿知 识和最新研究课题引入教学, 结合教材中相关知识点, 激发学生兴趣, 了解科学研究的前沿领域。 不定期安排演示实验, 讲解最新科研文献, 针对学生安排一些海报展示活动并评奖, 鼓励学生研 讨, 活跃课堂气氛。学生不仅锻炼了表达能力, 并且在提问中得到启发, 发现问题解决办法, 激发 学生科研创新热情和兴趣。同时, 非常重视分析化学实践教育, 将学生参加科研活动纳入教学计划, 设置科研学分。发达国家源源不断的科技创新能力正是来自于对学生的研究能力的培养。

\section{3 具体做法}

新工科教育的核心理念是理工学科交叉融合。分析化学是高等学校化学类专业的一门基础性课 程, 是构成化学类学生完整知识链的重要环节, 具有较强的实践性。在分析化学类课程体系建设中, 对分析化学专业课教学内容提出 “确保基础性、强化系统性、重视前沿交叉性和展现应用性” 的原 则, 从教学内容和教学方法两部分入手对分析化学类课程进行改革。工科专业与理科专业在课程设置、 培养目标上有很大差异, 理论课时少、实践环节多的特点在工科专业中非常鲜明。如何在有限的教 学学时内, 让学生既能系统牢固地掌握分析化学的基础理论, 又能了解到最新的分析化学前沿知 识, 同时具有熟练的实验操作技能, 是我们在教学中所面临的问题。课程内容的选择原则是什么, 哪些重点内容要讲, 哪些陈旧的内容不讲, 必须慎重。另外要体现工科特色, 理论联系有关专业实际, 联系社会热点和科研前沿问题, 如能源、生命、环境及材料等专业知识, 使学生达到学以致用, 让 学生从本科开始就初步接触科学研究, 培养科研创新意识, 为将来有意进一步读研的学生打好科研 创新能力的基础, 具有重要的现实意义和可操作性。 


\section{2 教学内容改革}

\section{1 精选课程内容、力求内容系统}

在哈尔滨工业大学的课程设置中, 分析化学类课程被安排在大学化学、无机化学课程之后开设。 在不影响课程系统性的前提下, 尽量少讲和不讲先期已学课程中的部分重复内容, 或引导学生进行 自学性复习总结, 避免低层次重复, 这样既节省了时间, 又可以介绍更多的分析化学领域中科研的 新内容新方法, 根据授课各专业的特色, 例如对于环境专业, 由于人类的频繁活动和不断攀升的能 源需求量, 全球二氧化碳的排放不断增加, 室温、常压可见光驱动光催化剂高效催化 $\mathrm{CO}_{2}$ 还原制甲烷 已成为研究热点, 需要使用气相色谱鉴定物质组分及组成。对于材料专业, 文献里研究材料的性质 会用到质谱、循环伏安、拉曼光谱, 以及原子力显微镜表征固体材料的表面特性。恰到好处地进行 学科知识的渗透、融合和拓展, 从而使整个分析化学类课程内容更加系统化, 在有限的课时内获得 更好的教学效果。

\section{2 密切贴近生活, 紧扣前沿进展}

借助网络信息, 综合运用多媒体素材, 特别是与新冠肺炎的检测方法、治疗方案、药物开发等 密切相关的信息和知识点, 例如结合国家卫生健康委员会发布的《新型冠状病毒肺炎诊疗方案(试行 第六版)》中的诊断标准, 作为素材来源融入分析化学教学过程。浅谈COVID-19新冠状病毒的核酸 检测方法, 血氧饱和度的现场快速检测, 从新冠肺炎的爆发看分析化学的重要性。例如, 为阻断病 毒传播, 医院、学校和车站等人流密集的公共场所都会使用 84 消毒液, 其中主要成分次氯酸钠的强 氧化性可杀菌消毒, 但次氯酸钠在光和热条件下易分解, 会降低有效氯含量, 减弱消杀效果。碘量 法是分析化学中重要的容量分析方法之一, 通常用于测定 84 消毒液中有效氯含量 ${ }^{[4]}$, 化学原理是次 氯酸盐在酸性介质中与碘离子反应, 生成单质碘; 用硫代硫酸钠 $\left(\mathrm{Na}_{2} \mathrm{~S}_{2} \mathrm{O}_{3}\right)$ 标准溶液滴定单质碘, 根 据终点时标准溶液的消耗量, 得到碘单质的量, 从而求得消毒液中的有效氯含量, 反应方程式如下:

$$
\begin{aligned}
& \mathrm{ClO}^{-}+2 \mathrm{I}^{-}+2 \mathrm{H}^{+}=\mathrm{I}_{2}+\mathrm{H}_{2} \mathrm{O}+\mathrm{Cl}^{-} \\
& \mathrm{I}_{2}+2 \mathrm{~S}_{2} \mathrm{O}_{3}^{2-}=2 \mathrm{I}^{-}+\mathrm{S}_{4} \mathrm{O}_{6}^{2-}
\end{aligned}
$$

滴定实验中要加入淀粉指示剂来判断滴定终点, 肉眼观察到溶液的颜色发生了突变, 立即停止 滴定, 根据 $\mathrm{Na}_{2} \mathrm{~S}_{2} \mathrm{O}_{3}$ 使用量计算出氯含量。同时可进一步提问, 引导学生对 “碘量法滴定终点为何出 现 “重蓝”现象” 展开讨论, 溶液中痕量的 $\mathrm{Fe}^{3+}$ 会促进 $\mathrm{I}^{-}$重新氧化生成 $\mathrm{I}_{2}$, 进而出现 “重蓝” 现象, 解决的方法是加入 $\mathrm{H}_{3} \mathrm{PO}_{4}$ 与 $\mathrm{Fe}^{3+}$ 络合 ${ }^{[5]}$ 。实验中自始至终都深刻体现现象和本质的辩证关系 ${ }^{[3]}$, 在课 堂上穿插讲述马克思主义哲学现象和本质的辩证法, 现象是事物的外在方面, 本质是事物的内在方 面, 滴定实验中, 颜色变化的内在本质是物质化学键的改变, 要透过现象来看本质, 坚持现象和本 质的辩证统一。同时, 将科研与教学有机结合促进教学质量提高。利用化学计量学辅助复杂波谱解 析的研究成果去改革分析化学课程的紫外吸收光谱分析、红外吸收光谱、质谱和色谱分析教学内容, 在不分离样本混合组分的情况下, 利用数学分离代替化学分离提取纯组分的光谱信息, 可以实现简 便、快速和准确的检测, 让学生了解分析化学解析方法的发展趋势以及一些交叉技术在分析中的应 用, 开拓学生的思维和视野, 提高了学生的学习兴趣。

\section{3 理论实践融合, 提高创新能力}

教学过程中, 应注重实验课程与理论课程知识点的融合互补, 吸收当代教学、科研中成熟的代 表性成果, 使理论课程与实验课程互相依托, 相辅相成, 鼓励学生主动发现科学问题和尝试解决问 题。例如在 2018年教学过程中, 讲解红外光谱仪器和原子力显微镜的工作原理知识时, 教师结合自 身 “原子层沉积制备氧化锌超疏水涂层材料” 的课题, 引导本科生申报了学校科技创新项目, 使用 红外光谱表征材料分子结构, 原子力显微镜观察涂层表面微观结构, 最终本科生以第二作者身份在 美国化学会国际知名杂志《应用表面界面》 $(\mathrm{IF}=8.758)$ 上发表了论文。课题实施过程中, 学生们团 结协作, 刻苦勤奋, 得到了很好的科研训练, 其中作为课题组长的本科生在毕业时被评为优秀毕业 生。另外一个例子是如何表征 $\mathrm{Al}$ 掺杂在 $\mathrm{ZnO}$ 薄膜中化学状态, 学生根据课程内容所学提出用红外光 
谱来证明, 查阅文献得知, 一般红外光谱波数范围 $400-4000 \mathrm{~cm}^{-1}$, 多是来表征有机物, 对于无机物, 需要使用远红外 $800 \mathrm{~cm}^{-1}$ 以下才能鉴别 $\mathrm{Zn}-\mathrm{O}$ 和 $\mathrm{Al}-\mathrm{O}$ 化合键, 通常 $466 \mathrm{~cm}^{-1}$ 和 $709 \mathrm{~cm}^{-1}$ 处分别归属 为 $\mathrm{Zn}-\mathrm{O}$ 键和 $\mathrm{Al}-\mathrm{O}$ 键的特征吸收峰 ${ }^{[6]}$ 。学生查到外地可以测试, 主动联系老师, 邮寄样品, 说清楚 测试要求, 最终成功得到有效数据, 本科生以第三作者发表了论文, 并成功获得美国加州伯克利大 学的奖学金继续攻读研究生。学生学到了分析化学仪器分析测试知识, 加强了分析解决问题能力, 锻炼了沟通交流能力, 更重要的是培养了学生对专业和科学研究的热爱。

\section{3 教学方法创新}

采用课堂启发自学式、重点强化式的教学方法, 实现教师为主导、学生为主体的双向互动 ${ }^{[7,8]}$ 。 将分析化学理论和日常生活中的一些现象紧密相联, 如选择性地介绍一些与实际生产以及人们衣、 食、住、行中有关的化学基本知识, 让学生感到分析化学其实离我们并不遥远, 从而激发学生的求 知欲望和学习兴趣。例如, 天然水中 $\mathrm{Ca} 、 \mathrm{Mg}$ 含量的测定, 阿司匹林片剂中乙酰水杨酸含量的测定, 如何测定奶粉中的含氮量, 如何鉴定奶粉中是否含有三聚氧胺等。另一方面, 开展兴趣教学, 将分 析化学领域学科发展史引入课堂。例如, 瑞典化学家阿伦尼乌斯提出了电离理论, 在被保守的教授 们虽之以鼻, 认为 “纯粹是空想, 无法相信” 的时候, 他坚持真理, 不断努力, 最终开创了电离学 说 ${ }^{[9]}$ 。通过小组互动讨论和分享查阅的资料, 充分锻炼学生的表达能力和文献检索能力, 了解书本 上的知识来源于科学家追求真理、持之以恒获得的智慧结晶。

\section{4 开放表界面材料分析化学实验室, 完善合理考核方式}

新工科的理念是多学科交叉与融合创新, 实现课堂教学与项目学习融合, 开放实验室, 创造新 机会、加强创新创业实践, 强调 “厚基础、强实践、重能力、求创新” 的新工科人才培养特色 ${ }^{[10]}$ 。 学生在获得一定的专业知识训练之后, 针对某一前沿课题开展项目研究, 利用科研设备转化为实践 育人平台, 将科研项目有取舍、有针对性地转化为教学实验, 提升实验条件和设备的能效, 使学科 建设成果和科研成果有效地转化为分析化学教育资源。例如将最新科研成果 “氨气诱变钻钛矿 $\mathrm{MAPbI}_{3}$ 能带结构” 转化为大学生创新实验, 结合所学知识, 让学生真正亲手操作使用紫外漫反射光 谱仪和红外光谱仪, 取得良好效果, 成果发表在清华大学主办的中文核心杂志《实验技术与管理》; 再比如 “3D打印” 和 “海水淡化” 科研成果选取部分转化为教学实验, 使得学生能够掌握3D打印原 理和操作, 并对目前光热材料在海水淡化研究中的应用有了充分的了解, 相关成果已经发表在化工 领域著名杂志Chem. Eng. J. 上, 增强了学生的科研创新意识。在燃烧热测定实验教学中, 我们从 “人 为什么要吃饭” 这一基本问题入手, 引导学生认识到能量过剩或缺乏带来的影响和结果, 从而去测 量日常所吃食物的热量, 将测量对象与日常生活相结合进行教学改革和创新, 实现了实验内容的 自主选择, 激起了学生的实验兴趣, 学生主动要求测量米饭、馒头、白菜、水果、干果等食品的热 量。此外, 介绍了一些分析类书籍, 如《我们身边的食品添加剂》, 让学生去阅读, 了解身边形形色 色的食品添加剂。初步研究表明, 将科研成果有目的、有选择性地转化为教学实验, 既能够促进科 研后备力量的储备, 也有利于大学生接触到最新科研成果, 激发学生学有所用、学以致用的成就感, 更加主动地充实自己, 以及继续攻读研究生的自信心。

\section{5 以学科竞赛及创新创业项目为载体, 激发学生创新精神}

组织学生积极参加校级和国家级竞赛活动, 比如 “祖光杯” 大赛、“挑战杯” 竞赛、“卓越杯” 化学实验竞赛, 新材料设计大赛、创新创业训练计划项目等。通过团队小组自主选题, 分工合作, 邀请经验丰富的教师和企业专家担任创新创业指导教师。例如, 通过分析化学实验学习锻炼学生实 验操作技能, 材料化学专业学生获得第五届 “卓越杯” 大学生化学新实验设计及化学实验技能竞赛 二等奖和三等奖; 来自卓越联盟九所高校近 200 名化学专业的本科生和 60 余位高校化学实验教学专 
家齐聚哈尔滨工业大学, 大连理工大学国家教学名师孟长功教授、天津大学国家级化学化工实验教 学示范中心唐向阳教授等十余位知名教授担任竞赛的评委。通过竞赛活动及创新创业项目的锻炼, 学生学会独立思考、发现、分析、解决问题, 获得创新思维和创造能力、团队合作和交际能力。再比 如, 为了实现创新思维深度交融、创业思想广泛汇聚、科研成果充分展示, 哈尔滨工业大学于 2018 年1月16-17日举办首届 “雄韬杯” 国际化学化工创新创业大赛, 大赛以 “化学创造美好生活” 为主 题, 涉及涵盖新能源、新材料、绿色化学化工、食品化学等方向, 共有国内外包括美国康奈尔大学、 乌克兰哈尔科夫国立大学、浙江大学、四川大学、吉林大学等 15 个学校的 22 支队伍参加, 论题涉及 化学、能源、材料等各个前沿领域, 不同专业的学生汇聚在一起进行 “论” 和 “谈”, 同时邀请专家、 教授进行交流互动, 提供了跨学科学习和研究的空间。

面对新技术、新业态、新产业快速发展和国家重大发展战略需求, 基于新工科背景, 积极探索 构建分析化学、仪器分析等分析化学类课程科研型教学模式, 结合团队教师 “空间用材料分析化学” 领域的科研成果, 探索科研项目转化为教学实验, 充分利用极端服役环境材料检测和表征的各种先 进测试仪器设备, 如红外光谱、紫外吸收光谱、原子力显微镜等, 实行科研反哺教学, 教学和科研 双轮驱动协同作用。通过学习, 引导学生积极参与科研项目, 培养学生、锻炼学生, 项目取得的经 验和成果为工科分析化学类人才培养提供可借鉴的指导作用。

\section{参 考 文 献}

[1] 吴爱华, 杨秋波, 郝杰. 高等工程教育, 2019, No. 1, 1.

[2] 戴可, 梁华, 黄鹤燕, 胡海龙, 霍冀川. 大学化学, 2019, 34 (4), 6 .

[3] 孙寅璐, 姜宏月, 熊英. 大学化学, 2021, 36 (3), 2002016.

[4] 谢芳, 鲍宇刚, 王传璧, 郭维麟. 中国消毒学杂志, 2003, 20 (3), 214.

[5] 李秀珍. 大学化学, 1988, 3 (4), 46.

[6] 杨小红, 童琴, 刘畅, 刘金库, 贺文智, 李光明. 物理化学学报, 2012, 28 (11), 2713.

[7] 吴硕, 刘志广, 宿艳, 王秀云, 刘哲, 张明旭. 中国大学教学, 2015, No. 1, 53 .

[8] 王玉枝, 旷亚非, 宦双燕, 郭慢丽, 王青. 化工高等教育, 2010, No. 2, 75.

[9] 间蒙钢, 慈洁琳. 大学化学, 2013, 28 (6), 71 .

[10] 徐晓飞, 沈毅, 钟诗胜, 姜永远, 张策. 高等工程教育研究, 2020, No. 2, 18. 